\title{
Colon Carcinoma Presenting as Streptococcus anginosus Bacteremia and Liver Abscess
}

\author{
Prashanth Rawla ${ }^{\mathrm{a}, \mathrm{e}}$, Anantha R. Vellipuram ${ }^{\mathrm{b}}$, Sathyajit S. Bandaru ${ }^{\mathrm{c}}$, \\ Jeffrey Pradeep Raj ${ }^{d}$
}

\begin{abstract}
A collection of dead white blood cells within the liver is called a liver abscess, and pyogenic liver abscess (PLA) is the most common type. PLA is commonly associated with biliary tract infections. However, in this case report, we present a rare cause of Streptococcus anginosus bacteremia and PLA which is associated with a carcinoma of the colon at the splenic flexure. The presentation mimicked a cholecystitis clinically, but the radio-imaging revealed a liver abscess. Blood cultures revealed an uncommon etiological agent, S. anginosus group which is otherwise a commensal in the human gut. In this case report, we aimed to summarize the microbiological aspects of $S$. anginosus group of organisms, the relevant clinicopathological considerations and the management.
\end{abstract}

Keywords: Colon carcinoma; Bacteremia; Streptococcus anginosus; Liver abscess

\section{Introduction}

A liver abscess is defined as a collection of dead white blood cells within the liver. Liver abscess may thus be classified into three based on the etiology, namely pyogenic (bacterial), amoebic and fungal liver abscess. Among the three types, pyogenic liver abscess (PLA) is the most common type, accounting for $80 \%$ of all cases [1]. In 1938, Ochsner et al published a review of 47 cases and concluded that surgical drainage is the definitive therapy for PLA, but mortality remained at $60-80 \%$ [2]. With the advent of newer radiological techniques, better

Manuscript submitted July 3, 2017, accepted September 5, 2017

a Department of Internal Medicine, Memorial Hospital of Martinsville and Henry County, VA, USA

${ }^{\mathrm{b}}$ Texas Tech University Health Sciences Center, El Paso, TX, USA

'Senior Research Associate, Beth Israel Deaconess Medical Center, Harvard Medical School, Boston, MA, USA

dDepartment of Pharmacology, St John's Medical College, Bangalore, India

${ }^{e}$ Corresponding Author: Prashanth Rawla, Department of Internal Medicine, Memorial Hospital of Martinsville and Henry County, VA, USA.

Email: rawlap@gmail.com

doi: https://doi.org/10.14740/gr884w microbiological isolation techniques and availability of antibiotics, the mortality has drastically come down to 5-30\% [1]. Here we present a case of Streptococcus anginosus bacteremia complicated by PLA secondary to colon cancer which is also a rarer risk factor for the development of PLA.

\section{Case Report}

A 62-year-old gentleman who is known to suffer from hypertension, dyslipidemia, and hypothyroidism for the past 15 years, presented to the emergency room with complaints of high-grade intermittent fever with chills but no associated rigors for the past 2 days. He also complained of minimal abdominal discomfort in the right upper quadrant and rated the pain at 2 on a pain scale of 10 . There was no radiation, no known aggravating or relieving factors associated with the pain. There was no history of any nausea, vomiting, diarrhea, other symptoms suggestive of urinary tract or respiratory tract infection. He was a chronic user of tobacco with a history smoking cigarettes amounting to 40 pack years over the last 40 years. He also consumes alcohol socially and gives no history of any intravenous (IV) drug abuse. His current medication history includes amlodipine 10 $\mathrm{mg}$ and lisinopril $40 \mathrm{mg}$ once daily for his hypertension, levothyroxine $50 \mu \mathrm{g}$ once daily for hypothyroidism and atorvastatin $20 \mathrm{mg}$ once daily at night for dyslipidemia.

On general physical examination, the patient had no pallor, icterus, lymphadenopathy or edema. He was running a temperature of $102^{\circ} \mathrm{F}\left(38.9^{\circ} \mathrm{C}\right)$ and was tachycardic with a heart rate of 110 beats per minute. His blood pressure on the right upper arm was measured at lying down posture as 90/60 $\mathrm{mm}$ Hg. Upon abdominal examination, there was mild tenderness in the right upper quadrant with no guarding or rebound tenderness. Cardiac examination revealed no murmurs and respiratory system examination was unremarkable.

A working diagnosis of cholecystitis was made at this juncture, and further investigations were proposed to confirm the diagnosis. The blood testing results are summarized in Table 1. There was leukocytosis with 70\% neutrophils, but liver function tests were normal. The patient underwent a CT abdomen with contrast. This identified an abscess in the right lobe of liver about $15 \mathrm{~mm}$ in diameter (Fig. 1) with no evidence of any gallstones or other abnormalities. An ultrasound scan of the right upper abdomen also reported the same findings and ruled out cholecystitis as there was no pericholecystic fluid found. Two 
Table 1. Laboratory Investigations on Day of Admission

\begin{tabular}{lll}
\hline Laboratory test (units) & Patient's value & Reference value \\
\hline White blood cells $\left(\mathrm{mm}^{3}\right)$ & 17,200 & $4,500-11,000$ \\
Platelets $\left(\mathrm{mm}^{3}\right)$ & 335,000 & $140,000-440,000$ \\
Alanine transferase (IU/L) & 33 & $10-53$ \\
Aspartate transferase (IU/L) & 27 & $10-53$ \\
Alkaline phosphatase (IU/L) & 142 & $45-115$ \\
C-reactive protein $(\mathrm{mg} / \mathrm{L})$ & 39 & $1-3$ \\
\hline
\end{tabular}

sets of blood cultures were sent, and he was started on empiric antibiotic treatment with IV vancomycin, metronidazole, and piperacillin-tazobactam. Meanwhile, all blood cultures grew Streptococcus anginosus, and the antibiotic treatment was deescalated to ceftriaxone $2 \mathrm{~g}$ once daily IV based on the sensitivity pattern. At this juncture, an opinion was sought from the interventional radiologist who opined that the abscess need not be drained as it was small and loculated, rather treat conservatively with IV antibiotics. Repeat blood cultures sent 2 days after starting antibiotic treatment turned out to be negative.

The patient was questioned further to investigate the possible source of infection except for a history of significant unexplained weight loss of about 15 pounds in the last 3 months. The patient then underwent a two-dimensional echocardiogram which ruled out any vegetation or valvular abnormalities. Trans-esophageal echocardiography was also normal. Meanwhile, the patient was discharged with a peripherally inserted central line to continue IV ceftriaxone for a total period of 4 weeks and to follow-up in the outpatient department (OPD) with colonoscopy. Subsequently, the following week, the patient had a colonoscopy done on OPD basis and was diagnosed to have colon carcinoma at the splenic flexure (Fig. 2). Biopsy of the colonic mass confirmed it as adenocarcinoma.

\section{Discussion}

The $S$. anginosus group belongs to the subgroup of viridans

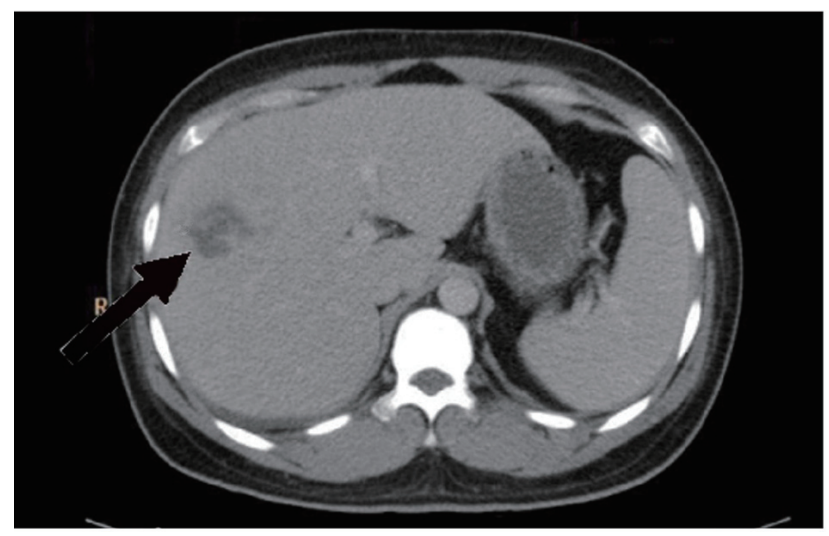

Figure 1. Computed tomography (CT) of the abdomen done at admission with intravenous contrast revealed a multiloculated thick-walled abscess in the right lobe of the liver (black arrow).

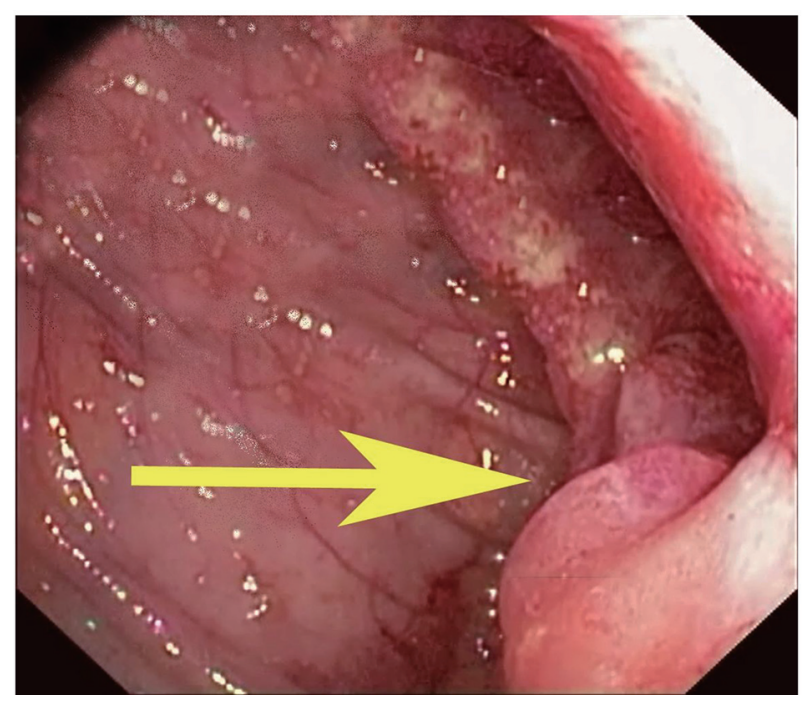

Figure 2. Colonoscopy revealed a mass (yellow arrow) at the splenic flexure which was confirmed to be an adenocarcinoma by biopsy.

Streptococci. This group consists of three distinct Streptococcal species, namely $S$. anginosus, $S$. intermedius and $S$. constellatus [3]. These organisms were first isolated by Guthof in 1956 from dental abscesses and are gram-positive, catalase-negative cocci. They are non-motile facultative anaerobes that may demonstrate alpha, beta or gamma hemolysis on blood agar [4]. The colony size on agar is typically less than $0.5 \mathrm{~mm}$ with a buttery butterscotch-like smell, and they demonstrate enhanced growth in the presence of carbon dioxide, while some of them need anaerobic conditions [5]. $S$. anginosus group is considered a part of the normal human flora mostly in the mouth, sinuses, throat, feces, and vagina. They rarely cause infection in a healthy individual [6]. The two most common places where the blood-mucosal barrier is breached due to a local infection are in the gastrointestinalpancreatico-hepatobiliary tracts and the thoracic cavity. These organisms are known for their tendency towards abscess formation. A case series with 51 patients reported that only six of them had associated abscesses and $53 \%$ of them had a local site of infection [7].

There is not much clinical need in distinguishing the members of the $S$. anginosus group. They usually present as polymicrobial infections [8]. The main pathogenesis in the formation of a deep seated abscess by the anginosus group is the production of an exotoxin by $S$. intermedius called intermedilysin. This is a cytolytic toxin which is specific for human cells especially for the hepatocytes [9]. They also produce hydrolytic enzymes which aid in liquefaction of pus and further spread of infection within the affected tissue [10]. It is also believed that interaction of the organism and polymorphonuclear cells may also play a role in the development of abscess formation [11]. In our patient, the colon cancer has resulted in the loss of integrity of the blood-mucosal barrier which has probably led to the $S$. anginosus bacteremia which is otherwise a normal commensal of the gut. Further to this, since the portal circulation is the first capillary bed, these organisms have accumulated in the liver parenchyma leading to an abscess [12]. 
The management approach of positive blood culture with $S$. anginosus group warrants a thorough search for an underlying deep abscess. In our patient, liver abscess was picked up prominently by the radio-imaging. The management of an abscess warrants surgical draining and debridement with intravenous antibiotics. If the abscess is small or there are multiple abscesses where draining is not practical, prolonged antimicrobial therapy as long as $6-8$ weeks is indicated [13]. Our patient was thus discharged with IV ceftriaxone based on the culture sensitivity report to be followed up weekly to assess the clinical improvement and was further referred to specialty treatment for the colon cancer.

Association of colorectal carcinoma with few microbial agents like group D streptococcus has been well implicated, though evidence as an etiological agent is contradictory. Similarly, there exists a serious doubt whether such an association exists between $S$. anginosus and colorectal carcinoma. Few case reports with similar association have already been published by Lin et al [14], Tzur et al [15], Millichap et al [16] and Masood et al [17]. Also, S. anginosus bacteremia has also been reported in patients with esophageal and gastric carcinoma [18].

\section{Conclusion}

Though $S$. anginosus group of organisms is a common commensal in the human gut, they are known to cause bacteremia when the blood mucosal integrity is lost. Bacteremia should warrant a thorough check for any underlying deep seated abscess as this group of Streptococci are known to cause abscesses, unlike the other Streptococci. Bacteremia is managed through IV antibiotics until clinical symptoms improve. Abscesses are usually treated surgically along with IV antibiotics. However, if draining is not possible, the management is conservative with prolonged IV antibiotics. Besides treatment of the abscess and bacteremia, the underlying cause is appropriately treated. Further epidemiological studies are warranted to identify if there is any association between $S$. anginosus bacteremia and colorectal carcinoma. Further studies are needed to find out if screening colonoscopy in patients with $S$. anginosus bacteremia is warranted.

\section{Funding}

This research received no specific grant from any funding agency in the public, commercial, or not-for-profit sectors.

\section{Conflict of Interest}

The authors do not have any conflict of interest to disclose.

\section{Financial Support}

None.

\section{Author Contributions}

Study design, drafting, critical revisions and final approval by PR, ARV, SSB and JPR.

\section{References}

1. Othman N, Mohamed Z, Yahya MM, Leow VM, Lim BH, Noordin R. Entamoeba histolytica antigenic protein detected in pus aspirates from patients with amoebic liver abscess. Exp Parasitol. 2013;134(4):504-510.

2. Ochsner A, DeBakey M, Murray S. Pyogenic Abscess of the Liver II. An Analysis of Forty-Seven Cases with Review of the Literature. Am J Surg. 1938;XL:292-319.

3. Claridge JE, 3rd, Attorri S, Musher DM, Hebert J, Dunbar S. Streptococcus intermedius, Streptococcus constellatus, and Streptococcus anginosus ("Streptococcus milleri group") are of different clinical importance and are not equally associated with abscess. Clin Infect Dis. 2001;32(10):1511-1515.

4. Spellerberg B, Brandt C. Streptococcus. In: Murray PR, Baron EJ, Jorgenson JH, et al (Eds). Manual of Clinical Microbiology, 9th ed, ASM Press: Washington, DC. 2009. Vol 1, p. 412.

5. Poole PM, Wilson G. Infection with minute-colonyforming beta-haemolytic streptococci. J Clin Pathol. 1976;29(8):740-745.

6. Yilmaz H, Yilmaz E, Karadag A, Esen S, Sunbul M, Leblebicioglu H. Liver abscess associated with an oral flora bacterium Streptococcus anginosus. Journal of Microbiology and Infectious Diseases. 2015;2(1):33-35.

7. Bert F, Bariou-Lancelin M, Lambert-Zechovsky N. Clinical significance of bacteremia involving the "Streptococcus milleri" group: 51 cases and review. Clin Infect Dis. 1998;27(2):385-387.

8. Toyoda K, Kusano N, Saito A. Pathogenicity of the Streptococcus milleri group in pulmonary infections--effect on phagocytic killing by human polymorphonuclear neutrophils. Kansenshogaku Zasshi. 1995;69(3):308-315.

9. Nagamune H, Whiley RA, Goto T, Inai Y, Maeda T, Hardie JM, Kourai H. Distribution of the intermedilysin gene among the anginosus group streptococci and correlation between intermedilysin production and deep-seated infection with Streptococcus intermedius. J Clin Microbiol. 2000;38(1):220-226.

10. Jacobs JA, Stobberingh EE. Hydrolytic enzymes of Streptococcus anginosus, Streptococcus constellatus and Streptococcus intermedius in relation to infection. Eur J Clin Microbiol Infect Dis. 1995;14(9):818-820.

11. Wanahita A, Goldsmith EA, Musher DM, Clarridge JE, 3rd, Rubio J, Krishnan B, Trial J. Interaction between human polymorphonuclear leukocytes and Streptococcus milleri group bacteria. J Infect Dis. 2002;185(1):85-90.

12. Boleij A, van Gelder MM, Swinkels DW, Tjalsma H. Clinical Importance of Streptococcus gallolyticus infection among colorectal cancer patients: systematic review and meta-analysis. Clin Infect Dis. 2011;53(9):870-878. 
13. Corredoira J, Casariego E, Moreno C, Villanueva L, Lopez, Varela J, Rodriguez A, et al. Prospective study of Streptococcus milleri hepatic abscess. Eur J Clin Microbiol Infect Dis. 1998;17(8):556-560.

14. Lin CY, Chao PC, Hong GJ, Tsai YT, Lee CY, Tsai CS. Infective endocarditis from Streptococcus viridans associated with colonic carcinoma: a case report. J Card Surg. 2008;23(3):263-265.

15. Tzur T, Liberman S, Felzenstein I, Cohen R, Rivkind AI, Almogy G. Liver abscesses caused by Streptococcus milleri: an uncommon presenting sign of silent colonic cancer. Isr Med Assoc J. 2003;5(3):206-207.
16. Millichap JJ, McKendrick AI, Drelichman VS. Streptococcus intermedius liver abscesses and colon cancer: a case report. West Indian Med J. 2005;54(5):341-342.

17. Masood U, Sharma A, Lowe D, Khan R, Manocha D. Colorectal cancer associated with streptococcus anginosus bacteremia and liver abscesses. Case Rep Gastroenterol. 2016;10(3):769-774.

18. Sasaki H, Ishizuka T, Muto M, Nezu M, Nakanishi Y, Inagaki Y, Watanabe $\mathrm{H}$, et al. Presence of Streptococcus anginosus DNA in esophageal cancer, dysplasia of esophagus, and gastric cancer. Cancer Res. 1998;58(14):29912995. 